\title{
Trends in Utilization and Outcomes of Pulmonary Artery Catheterization in Heart Failure With and Without Cardiogenic Shock
}

\author{
GABRIEL. A. HERNANDEZ, MD,${ }^{1,5}$ ALEJANDRO LEMOR, MD, ${ }^{2,3}$ VANESSA BLUMER, MD, ${ }^{4}$ CARLOS A. RUEDA, MD ${ }^{5}$ \\ SANDIP ZALAWADIYA, MBBS, ${ }^{5}$ LYNNE W. STEVENSON, MD, ${ }^{5}$ AND JOANN LINDENFELD, MD $^{5}$
}

Miami, Florida; Detroit, Michigan; Lima, Peru; and Nashville, Tennessee

\begin{abstract}
Background: The pulmonary artery catheter (PAC) has been used in a wide range of critically ill patients. It is not indicated for routine care of heart failure (HF), but its role in cardiogenic shock (CS) has not been clarified. Methods and Results: We conducted a retrospective cohort study with the use of the National Inpatient Sample and identified a total of 9,431,944 adult patients admitted from 2004 to 2014 with the primary diagnosis of HF $(n=8,516,528)$ or who developed CS $(n=915,416)$ during the index hospitalization. Overall, patients with PAC had increased hospital costs, length of stay, and mechanical circulatory support use. In patients with $\mathrm{HF}$, PAC use was associated with higher mortality $(9.9 \%$ vs $3.3 \%$, OR $3.96 ; P<.001)$ but the excess of mortality declined over time. In those with CS, PAC was associated with lower mortality (35.1\% vs $39.2 \%$, OR $0.91 ; P<.001)$ and in-hospital cardiac arrest $(14.9 \%$ vs $18.3 \%$, OR $0.77 ; P<.001)$; this paradox persisted after propensity score matching.

Conclusions: The use of PAC in CS has decreased from 2004 to 2014, although its use is now associated with improved outcomes, which may reflect better selection of patients or better use of the information to guide therapies. Our data provide reassurance that PAC use in this population is an appropriate strategy. (J Cardiac Fail 2019;25:364-371)
\end{abstract}

Key Words: Heart failure, cardiogenic shock, pulmonary artery catheter.

For more than 50 years, the pulmonary artery catheter (PAC) has been used in clinical practice, and may have been overused in a wide range of critically ill patients. ${ }^{1,2}$ During the past decades, there has been a notable decline in its use ${ }^{3}$ after meta-analyses and randomized trials reported neutral effects on clinical outcomes in these patients. ${ }^{2,4,5}$ Nevertheless, advocates of PAC monitoring have emphasized its potential role for triage and guidance of therapy in patients presenting with circulatory decompensation.

From the ${ }^{1}$ Division of Cardiology, Department of Medicine, University of Miami Miller School of Medicine, Miami, Florida; ${ }^{2}$ Division of Cardiology, Department of Medicine, Henry Ford Hospital, Detroit, Michigan; ${ }^{3}$ Centro de Investigación de Epidemiología Clínica y Medicina Basada en Evidencias, Facultad de Medicina Humana, Universidad de San Martín de Porres, Lima, Peru; ${ }^{4}$ Internal Medicine, Department of Medicine, University of Miami Miller School of Medicine, Miami, Florida and ${ }^{5}$ Division of Advanced Heart Failure and Transplant Cardiology, Vanderbilt University Medical Center, Nashville, Tennessee.

Manuscript received November 7, 2018; revised manuscript received February 8, 2019; revised manuscript accepted March 4, 2019.

Reprint requests: JoAnn Lindenfeld, MD, Professor of Medicine, Director, Heart Failure and Transplantation Section, Vanderbilt Heart and Vascular Institute, 1215 21st Ave, South, South Tower-5209, Nashville, TN 37232-8802. Tel: 615-936-8187; Fax: 615-936-1643. E-mail:

amandavest@hotmail.co.uk

See page 370 for disclosure information.

$1071-9164 / \$$ - see front matter

(C) 2019 Elsevier Inc. All rights reserved.

https://doi.org/10.1016/j.cardfail.2019.03.004
These neutral results of PAC use may reflect challenges in patient selection (imposed by the requirement for equipoise before randomization), lack of specific therapeutic algorithms in response to information supplied by the PAC, poor provider data interpretation, lack of beneficial therapies to apply, or a detrimental effect of the PAC itself. The Evaluation Study of Congestive Heart Failure and Pulmonary Artery Catheterization Effectiveness (ESCAPE) trial ${ }^{2}$ demonstrated that PACs are not routinely indicated in hospitalized patients with chronic decompensated heart failure (HF). There have been major advances in the acute management of HF, in both medical and device therapies, since the ESCAPE trial was released. Moreover, development of mechanical circulatory support (MCS) therapy has become widely available for cardiogenic shock (CS) patients, yet there have been no randomized trials evaluating the benefit of PAC in this population. We hypothesized that invasive monitoring may improve outcomes in patients with CS in the contemporary era.

\section{Methods}

\section{Data Source}

We conducted a retrospective cohort study with the use of the National Inpatient Sample (NIS) database from 2004 to 2014. The NIS is a subset of the Healthcare Cost and Utilization Project sponsored by the Agency for Healthcare 
Research and Quality and is the largest publicly available allpayer inpatient care database in the United States. It was designed as a stratified probability sample to be representative of all nonfederal acute-care hospitals. Up to 30 discharge diagnoses and 15 procedures are recorded for each patient with the use of the International Classification of Diseases, 9th Revision, Clinical Modification (ICD-9-CM) codes.

Chi-square and Wilcoxon rank sum tests were used to compare proportions and continuous variables for the differences in the baseline characteristics of patients. Multivariate linear and logistic regression models were created to compare continuous and categoric outcomes among our study groups. Poisson regression was used to evaluate length of stay. Using backward elimination and variables with $P$ values $<.2$, we generated a model adjusted for potential recorded confounders (age, sex, race/ethnicity, comorbidities [estimated by Charlson comorbidity index (CCI)], hospital location [rural or urban], geographic region, hospital teaching status, and hospital bed size).

With the intention of controlling for the differences in baseline patient and hospital characteristics, we used propensity score matching. The propensity score was calculated with the use of a multivariate logistic regression model for different variables that included patient demographics, comorbidities, and hospital characteristics; then we matched all patients using a one-to-one scheme without replacement using the nearest-number neighbor algorithm with a maximum caliber of 0.01 of the propensity score. We reported standardized differences between the 2 groups to assess for a successful match, with results of $<10 \%$ to determine an adequate match. Using the matched cohort, we used a univariate logistic and linear regression to analyze statistical significance between our outcomes in both groups, results are reported as odds ratios (ORs) with 95\% confidence intervals (CIs).

\section{Study Sample}

We identified all adult patients admitted with a primary diagnosis of HF (ICD-9-CM codes 428.x, 402.01, 402.11, 402.91, 404.01, 404.03, 404.11, 404.13, 404.91, and 404.93). In addition, to gather all patients with CS, we included those with the ICD-9-CM code 785.51 listed as a hospital diagnosis. We then separated our population into 2 mutually exclusive cohorts: (1) CS cohort ("CS"), which included all patients who were coded with ICD-9-CM code 785.51 regardless of whether they had prior HF or if they were admitted with the aforementioned HF codes, and (2) HF without CS cohort ("HF"), which included all patients with HF codes without a discharge ICD-9-CM code 785.51.

We identified those patients who underwent invasive hemodynamic monitoring with the use of the ICD-9 procedure codes 89.63 (pulmonary artery pressure monitoring), 89.64 (pulmonary artery wedge monitoring), 89.66 (measurement of mixed venous blood gases), 89.67 (monitoring of cardiac output by oxygen consumption technique [Fick method]), and 89.68 (monitoring of cardiac output by other technique [thermodilution indicator]). Previous studies have used similar methodology to evaluate trends and outcomes of PAC with the use of the NIS ${ }^{3,6,7}$ and Medicare inpatient claims databases. ${ }^{8}$ We excluded the procedure code 37.21 (right heart catheterization) because this code is intended to be used in the catheterization laboratory ("in-and-out" procedure), which may be an isolated measurement or part of a left heart catheterization procedure. Patients were excluded if they were $<18$ years of age and if they had missing mortality data. We subdivided patients with HF and CS based on the presence or absence of PAC.

\section{Outcomes}

The primary outcome was in-hospital mortality, which was provided within the NIS for each discharge. Secondary outcomes included the length of hospital stay (LOS), cardiac arrest, MCS use, and hospital costs.

\section{Results}

We included a weighted total of 9,431,944 patients hospitalized with the primary diagnosis of HF $(n=8,516,528)$ and CS $(n=915,416)$ during the index hospitalization (Supplemental Fig. 1). Overall, patients with CS were younger than those with $\mathrm{HF}(68 \pm 15$ vs $72 \pm 14 \mathrm{y})$. A PAC was used in $0.87 \%$ of all patients admitted with $\mathrm{HF}(\mathrm{n}=74,205)$ and in $8.7 \%$ of those with CS $(n=79,682)$. However, patients with CS represented $51 \%$ of all PAC used. The PAC was most commonly used in large and/or teaching hospitals. Table 1 presents patients and hospital characteristics in detail.

In patients admitted with $\mathrm{HF}$ without CS, those with PAC were younger ( $63 \pm 15$ vs $73 \pm 14 \mathrm{y})$ and more commonly male (63\% vs $49.4 \%$ ) compared with those without PAC $(P<.001)$. HF patients with PAC were less likely to have preexisting hypertension, diabetes, chronic obstructive pulmonary disease (COPD), or end-stage renal disease (ESRD) and had a lower burden of comorbidities as estimated by CCI. A PAC was more frequently used in patients with electrolyte disorder, atrial fibrillation, and prior implantable cardiac defibrillator. History of myocardial infarction and percutaneous coronary revascularization was similar for both groups.

In patients with CS, those with PAC were also younger than those without PAC $(64 \pm 15$ vs $68 \pm 14 \mathrm{y} ; P<.001)$. These patients were also more commonly male $(63.8 \%$ vs $59.3 \%$ ), with the largest percentage admitted with STelevation myocardial infarction (STEMI; 24.3\%), followed by non-STEMI $(12.9 \%)$, acute HF (11.4\%), and sepsis (9.3\%). Supplemental Table 2 presents the primary discharge diagnosis for patients in the CS cohort. These patients were less likely to have preexisting hypertension, ESRD, or COPD; however, the presence of diabetes, anemia, liver disease, and CCI were similar for patients with or without PAC. Similarly to the HF patients, history of myocardial infarction and percutaneous coronary intervention was similar in the presence or absence of PAC. 
Table 1. Baseline Patient and Hospital Characteristics

\begin{tabular}{|c|c|c|c|c|c|c|c|c|}
\hline \multirow[b]{2}{*}{ Charracteristic } & \multicolumn{4}{|c|}{ Cardiogenic Shock } & \multicolumn{4}{|c|}{ Heart Failure } \\
\hline & Overall & PAC & No PAC & $P$ Value & Overall & PAC & No PAC & $P$ Value \\
\hline No. of patients & 915,416 & 79,682 & 835,734 & & $8,516,528$ & 74,205 & $8,442,323$ & \\
\hline Mean age, y (SD) & $67.8(14.5)$ & $64.0(14.7)$ & $68.1(14.4)$ & $<.001$ & $72.8(14.3)$ & $63.1(14.8)$ & $72.9(14.2)$ & $<.001$ \\
\hline $18-34$ y & $10.6 \%$ & $15.3 \%$ & $10.2 \%$ & & $1.1 \%$ & $4.6 \%$ & $1.1 \%$ & \\
\hline $35-54 \mathrm{y}$ & $30.5 \%$ & $35.3 \%$ & $30.0 \%$ & & $11.1 \%$ & $21.4 \%$ & $11.0 \%$ & \\
\hline $55-74 \mathrm{y}$ & $37.6 \%$ & $36.3 \%$ & $37.7 \%$ & & $35.5 \%$ & $49.5 \%$ & $35.4 \%$ & \\
\hline$>75 \mathrm{y}$ & $21.3 \%$ & $13.1 \%$ & $22.1 \%$ & & $52.3 \%$ & $24.6 \%$ & $52.5 \%$ & \\
\hline \multicolumn{9}{|l|}{ Sex } \\
\hline Female & $40.3 \%$ & $36.2 \%$ & $40.7 \%$ & $<.001$ & $50.4 \%$ & $37.0 \%$ & $50.6 \%$ & $<.001$ \\
\hline Male & $59.7 \%$ & $63.8 \%$ & $59.3 \%$ & & $49.6 \%$ & $63.0 \%$ & $49.4 \%$ & \\
\hline \multicolumn{9}{|l|}{ Race } \\
\hline White & $72.7 \%$ & $71.4 \%$ & $72.8 \%$ & $<.001$ & $69.1 \%$ & $65.7 \%$ & $69.1 \%$ & $<.001$ \\
\hline Black & $12.0 \%$ & $12.3 \%$ & $12.0 \%$ & & $18.7 \%$ & $20.7 \%$ & $18.6 \%$ & \\
\hline Hispanic & $8.0 \%$ & $8.0 \%$ & $8.0 \%$ & & $7.8 \%$ & $7.9 \%$ & $7.8 \%$ & \\
\hline Asian or Pacific Islander & $3.0 \%$ & $3.4 \%$ & $3.0 \%$ & & $1.7 \%$ & $2.0 \%$ & $1.7 \%$ & \\
\hline Other & $4.4 \%$ & $5.0 \%$ & $4.3 \%$ & & $2.8 \%$ & $3.7 \%$ & $2.8 \%$ & \\
\hline \multicolumn{9}{|l|}{ Comorbidities } \\
\hline Hypertension & $51.6 \%$ & $48.0 \%$ & $51.9 \%$ & $<.001$ & $68.9 \%$ & $55.9 \%$ & $69.0 \%$ & $<.001$ \\
\hline Diabetes mellitus & $31.5 \%$ & $31.7 \%$ & $31.5 \%$ & .517 & $42.9 \%$ & $38.7 \%$ & $42.9 \%$ & $<.001$ \\
\hline End-stage renal disease & $5.8 \%$ & $3.8 \%$ & $6.0 \%$ & $<.001$ & $5.0 \%$ & $3.4 \%$ & $5.0 \%$ & $<.001$ \\
\hline Preexistent heart failure & $55.5 \%$ & $64.6 \%$ & $54.6 \%$ & $<.001$ & & & & \\
\hline Obesity (BMI $\geq 30 \mathrm{~kg} / \mathrm{m}^{2}$ ) & $10.5 \%$ & $11.3 \%$ & $10.4 \%$ & $<.001$ & $13.6 \%$ & $15.9 \%$ & $13.5 \%$ & $<.001$ \\
\hline Chronic pulmonary disease & $24.2 \%$ & $22.8 \%$ & $24.3 \%$ & $<.001$ & $37.1 \%$ & $28.3 \%$ & $37.2 \%$ & $<.001$ \\
\hline Peripheral vascular disease & $12.0 \%$ & $11.2 \%$ & $12.1 \%$ & .001 & $11.8 \%$ & $10.1 \%$ & $11.8 \%$ & $<.001$ \\
\hline Hypothyroidism & $9.5 \%$ & $8.8 \%$ & $9.6 \%$ & $<.001$ & $16.5 \%$ & $13.7 \%$ & $16.5 \%$ & $<.001$ \\
\hline Previous myocardial infarction & $8.2 \%$ & $8.1 \%$ & $8.2 \%$ & .903 & $12.6 \%$ & $12.7 \%$ & $12.6 \%$ & .534 \\
\hline Previous PCI & $6.6 \%$ & $6.5 \%$ & $6.6 \%$ & .471 & $8.3 \%$ & $7.8 \%$ & $8.3 \%$ & .029 \\
\hline Previous CABG & $6.1 \%$ & $5.5 \%$ & $6.2 \%$ & .001 & $15.0 \%$ & $11.1 \%$ & $15.1 \%$ & $<.001$ \\
\hline Previous stroke/TIA & $5.5 \%$ & $4.5 \%$ & $5.6 \%$ & $<.001$ & $7.1 \%$ & $5.1 \%$ & $7.1 \%$ & $<.001$ \\
\hline Known CAD & $46.9 \%$ & $44.7 \%$ & $47.1 \%$ & $<.001$ & $46.5 \%$ & $43.9 \%$ & $46.6 \%$ & $<.001$ \\
\hline Carotid artery disease & $1.3 \%$ & $1.3 \%$ & $1.3 \%$ & .931 & $1.2 \%$ & $1.4 \%$ & $1.2 \%$ & .018 \\
\hline Atrial fibrillation & $29.5 \%$ & $32.5 \%$ & $29.3 \%$ & $<.001$ & $36.5 \%$ & $38.2 \%$ & $36.5 \%$ & $<.001$ \\
\hline Prior cardiac defibrillator & $2.5 \%$ & $4.2 \%$ & $2.3 \%$ & $<.001$ & $7.6 \%$ & $17.6 \%$ & $7.5 \%$ & $<.001$ \\
\hline Prior pacemaker & $2.6 \%$ & $2.0 \%$ & $2.7 \%$ & $<.001$ & $9.5 \%$ & $4.9 \%$ & $9.5 \%$ & $<.001$ \\
\hline Anemia & $20.5 \%$ & $21.3 \%$ & $20.4 \%$ & .007 & $29.3 \%$ & $26.6 \%$ & $29.4 \%$ & $<.001$ \\
\hline Liver disease & $3.3 \%$ & $3.7 \%$ & $3.2 \%$ & .002 & $2.4 \%$ & $4.3 \%$ & $2.4 \%$ & $<.001$ \\
\hline Cancer & $4.5 \%$ & $3.5 \%$ & $4.6 \%$ & $<.001$ & $3.5 \%$ & $2.7 \%$ & $3.5 \%$ & $<.001$ \\
\hline Electrolyte disorders & $52.9 \%$ & $57.6 \%$ & $52.5 \%$ & $<.001$ & $25.8 \%$ & $39.2 \%$ & $25.7 \%$ & $<.001$ \\
\hline Other neurologic disorder & $7.8 \%$ & $6.1 \%$ & $7.9 \%$ & $<.001$ & $6.3 \%$ & $4.1 \%$ & $6.3 \%$ & $<.001$ \\
\hline \multicolumn{9}{|l|}{ Charlson comorbidity index } \\
\hline 0 & $25.0 \%$ & $25.0 \%$ & $25.0 \%$ & .010 & $22.0 \%$ & $21.4 \%$ & $22.0 \%$ & $<.001$ \\
\hline 1 & $21.3 \%$ & $21.9 \%$ & $21.2 \%$ & & $19.4 \%$ & $23.6 \%$ & $19.4 \%$ & \\
\hline 2 & $24.8 \%$ & $25.2 \%$ & $24.7 \%$ & & $26.3 \%$ & $24.2 \%$ & $26.3 \%$ & \\
\hline$\geq 3$ & $29.0 \%$ & $27.9 \%$ & $29.1 \%$ & & $32.2 \%$ & $20.9 \%$ & $32.3 \%$ & \\
\hline \multicolumn{9}{|l|}{ Other characteristics } \\
\hline Teaching hospital & $58.3 \%$ & $72.2 \%$ & $57.0 \%$ & $<.001$ & $41.5 \%$ & $71.2 \%$ & $41.2 \%$ & $<.001$ \\
\hline Elective admission & $11.7 \%$ & $14.1 \%$ & $11.4 \%$ & $<.001$ & $8.9 \%$ & $18.0 \%$ & $8.9 \%$ & $<.001$ \\
\hline \multicolumn{9}{|l|}{ Median household income } \\
\hline US\$ $1-39,999$ & $28.4 \%$ & $25.9 \%$ & $28.7 \%$ & $<.001$ & $32.8 \%$ & $31.9 \%$ & $32.9 \%$ & $<.001$ \\
\hline US\$ $40,000-50,999$ & $26.3 \%$ & $24.6 \%$ & $26.5 \%$ & & $26.8 \%$ & $25.0 \%$ & $26.9 \%$ & \\
\hline US\$ 51, 000-65,999 & $24.0 \%$ & $25.6 \%$ & $23.9 \%$ & & $22.5 \%$ & $23.5 \%$ & $22.5 \%$ & \\
\hline $\mathrm{US} \$ \geq 66,000$ & $21.2 \%$ & $24.0 \%$ & $20.9 \%$ & & $17.8 \%$ & $19.6 \%$ & $17.8 \%$ & \\
\hline \multicolumn{9}{|l|}{ Primary payer } \\
\hline Medicare/Medicaid & $70.9 \%$ & $65.8 \%$ & $71.4 \%$ & $<.001$ & $82.9 \%$ & $69.5 \%$ & $83.0 \%$ & $<.001$ \\
\hline Private insurance & $21.5 \%$ & $26.5 \%$ & $21.0 \%$ & & $12.0 \%$ & $24.3 \%$ & $11.9 \%$ & \\
\hline Self-pay/other & $7.6 \%$ & $7.8 \%$ & $7.6 \%$ & & $5.2 \%$ & $6.2 \%$ & $5.1 \%$ & \\
\hline \multicolumn{9}{|l|}{ Discharge disposition } \\
\hline Home & $75.0 \%$ & $79.4 \%$ & $75.0 \%$ & $<.001$ & $53.7 \%$ & $54.9 \%$ & $53.6 \%$ & $<.001$ \\
\hline Nursing home/facility & $20.8 \%$ & $15.0 \%$ & $20.8 \%$ & & $35.5 \%$ & $32.4 \%$ & $35.8 \%$ & \\
\hline Against medical advice & $1.1 \%$ & $0.5 \%$ & $1.1 \%$ & & $0.5 \%$ & $0.5 \%$ & $0.5 \%$ & \\
\hline Transfer to another hospital & $3.1 \%$ & $5.2 \%$ & $3.1 \%$ & & $10.3 \%$ & $12.3 \%$ & $10.1 \%$ & \\
\hline
\end{tabular}

BMI, Body mass index; CABG, coronary artery bypass graft; CAD, coronary artery disease; PCI, percutaneous coronary intervention; TIA, transient ischemic attack.

\section{Outcomes}

The use of PAC in patients with HF without CS was associated with a higher prevalence of in-hospital mortality
(9.9\% vs $3.3 \%$, OR 3.96, 95\% CI 3.68-4.26; $P<.001$ ), whereas its use in patients with CS was associated with a lower mortality $(35.1 \%$ vs $39.2 \%$, OR $0.91,95 \%$ CI $0.88-0.95 ; \quad P<.001)$. This paradox persisted after 
propensity score matching with a mortality rate of $6.7 \%$ vs $2.4 \%$ (OR 2.95, CI 2.56-3.39; $P<.001$ ) in patients with HF without CS with and without PCA, respectively, and $34.9 \%$ vs $37 \%$ (OR 0.91 , CI $0.87-0.97 ; P=.001$ ) for CS patients with and without PAC, respectively. In-hospital cardiac arrest was more frequently seen in those with $\mathrm{HF}$ receiving a PAC compared with those with $\mathrm{HF}$ but no PAC (2.7\% vs $0.6 \%$, OR 3.96, CI 3.51-4.48; $P<.001$ ). In patients with CS, PAC was associated with lower rates of in-hospital cardiac arrest compared with those with CS and no PAC (14.9\% vs $18.3 \%$, OR 0.77 , CI $0.74-0.81$; $P<.001$; Table 2 and 3$)$.

In patients with $\mathrm{HF}$ without $\mathrm{CS}$, PAC was much more likely to be used in those who developed acute respiratory failure (OR 2.10, CI 1.99-2.21; $P<.001$ ) or acute kidney injury (AKI; OR 2.97, CI 2.85-3.09; $P<.001$ ) or required mechanical ventilation (OR 6.15, CI 5.79-6.53; $P<.001$ ) or hemodialysis (OR 3.47, CI 3.15-3.81; $P<.001$ ). The association with acute respiratory failure (OR 1.13, CI 1.09-1.17; $P<.001$ ), mechanical ventilation (OR 1.26,

Table 2. In-Hospital Outcomes for Acute Decompensated Heart Failure and Cardiogenic Shock

\begin{tabular}{|c|c|c|c|c|c|c|c|c|c|c|}
\hline \multirow[b]{2}{*}{ Outcome } & \multicolumn{5}{|c|}{ Cardiogenic Shock } & \multicolumn{5}{|c|}{ Heart Failure } \\
\hline & Overall & PAC & No PAC & OR $(95 \% \mathrm{CI})^{*}$ & $P$ Value & Overall & PAC & No PAC & OR $(95 \% \mathrm{CI})^{*}$ & $P$ Value \\
\hline $\operatorname{LOS} \geq 5 \mathrm{~d}$ & $66.2 \%$ & $80.4 \%$ & $64.9 \%$ & $2.03(1.94-2.12)$ & $<.001$ & $41.7 \%$ & $78.2 \%$ & $41.4 \%$ & $5.46(5.21-5.73)$ & $<.001$ \\
\hline CS use & $26.2 \%$ & $38.4 \%$ & $25.0 \%$ & $1.82(1.75-1.89)$ & $<.001$ & $0.3 \%$ & $7.3 \%$ & $0.2 \%$ & $18.19(16.92-19.56)$ & $<.001$ \\
\hline AKI requiring dialysis & $6.1 \%$ & $9.6 \%$ & $5.8 \%$ & $1.52(1.43-1.62)$ & $<.001$ & $0.9 \%$ & $4.7 \%$ & $0.9 \%$ & $3.47(3.15-3.81)$ & $<.001$ \\
\hline Acute kidney injury & $46.8 \%$ & $55.8 \%$ & $45.9 \%$ & $1.47(1.42-1.53)$ & $<.001$ & $15.6 \%$ & $37.0 \%$ & $15.4 \%$ & $2.97(2.85-3.09)$ & $<.001$ \\
\hline & $.2 \%$ & $26.9 \%$ & $.7 \%$ & $1.36(1.31-1.42)$ & 001 & & $14.6 \%$ & $6.4 \%$ & $2.75(2.60-2.90)$ & $<.001$ \\
\hline Intubation & $50.1 \%$ & $56.3 \%$ & $49.5 \%$ & $1.26(1.22-1.31)$ & $<.001$ & $2.1 \%$ & $14.4 \%$ & $2.0 \%$ & $6.15(5.79-6$. & $<.001$ \\
\hline Vascular complications & $.5 \%$ & $19.0 \%$ & & $1.15(1.10-1.20)$ & $<.001$ & $7.3 \%$ & $19.0 \%$ & $7.2 \%$ & 3.30( & $<.001$ \\
\hline Acute respiratory failure & $42.7 \%$ & $45.7 \%$ & $42.5 \%$ & $1.13(1.09-1.17)$ & $<.001$ & $7.5 \%$ & $16.8 \%$ & $7.4 \%$ & $2.10(1.99-2.21)$ & $<.001$ \\
\hline Major bleeding & $7.7 \%$ & $7.7 \%$ & $7.7 \%$ & $1.00(0.93-1.07)$ & .947 & $2.2 \%$ & $3.9 \%$ & $2.2 \%$ & $2.07(1.89-2.28)$ & $<.001$ \\
\hline Mortality & $38.8 \%$ & $35.1 \%$ & $39.2 \%$ & $0.91(0.88-0.95)$ & & $3.3 \%$ & $9.9 \%$ & $3.3 \%$ & $3.96(3.68-4.26)$ & $<.001$ \\
\hline CI & $.6 \%$ & $18.4 \%$ & $23.0 \%$ & $0.79(0.76-0.83)$ & $<.001$ & $0.9 \%$ & $2.4 \%$ & $0.9 \%$ & $.85-2.37)$ & $<.001$ \\
\hline Cardiac arrest & $18.0 \%$ & $14.9 \%$ & $18.3 \%$ & $0.77(0.74-0.81)$ & $<.001$ & $0.6 \%$ & $2.7 \%$ & $0.6 \%$ & $3.96(3.51-4.48)$ & $<.001$ \\
\hline Median LOS, d (IQR) & $7(3-14)$ & $12(6-20)$ & $7(3-14)$ & - & $<.001$ & $4(2-6)$ & $9(5-16)$ & $4(2-6)$ & - & $<.001$ \\
\hline Median hospital costs & $\$ 29,927$ & $\$ 49,873$ & $\$ 28,286$ & - & $<.001$ & $\$ 7,136$ & $\$ 23,266$ & $\$ 7,091$ & - & $<.001$ \\
\hline Coronary bypass surgery & $12.4 \%$ & $14.4 \%$ & $12.2 \%$ & $2.88(1.50-3.31)$ & $<.001$ & $0.3 \%$ & $3.3 \%$ & $0.3 \%$ & $7.60(6.72-8.60)$ & $<.001$ \\
\hline & & & & $3.42(3.11-3.78)$ & & $0.1 \%$ & $4.5 \%$ & $0.1 \%$ & $19.04(17.20-21.08)$ & $<.001$ \\
\hline Heart transplantation & $0.6 \%$ & $2.0 \%$ & $0.5 \%$ & $1.12(1.06-1.18)$ & $<.001$ & $0.1 \%$ & $2.7 \%$ & $0.1 \%$ & $13.24(11.53-15.20)$ & $<.001$ \\
\hline
\end{tabular}

AKI, acute kidney injury; IQR, interquartile range; LVAD, left ventricular assist device; LOS, length of stay; MCS, mechanical circulatory support; PCI, percutaneous coronary intervention.

*Adjusted for age, sex, race, Charlson comorbidity index, hospital teaching status, hospital region, hospital bed size, and elective admission.

Table 3. In-Hospital Outcomes After Propensity Score Matching

\begin{tabular}{|c|c|c|c|c|c|c|c|c|}
\hline \multirow[b]{2}{*}{ Outcome } & \multicolumn{4}{|c|}{ Cardiogenic Shock } & \multicolumn{4}{|c|}{ Heart Failure } \\
\hline & $\begin{array}{c}\text { PAC } \\
(\mathrm{n}=11,139)\end{array}$ & $\begin{array}{c}\text { No PAC } \\
(\mathrm{n}=11,139)\end{array}$ & OR $(95 \% \mathrm{CI})^{*}$ & $P$ Value & $\begin{array}{c}\text { PAC } \\
(\mathrm{n}=11,640)\end{array}$ & $\begin{array}{c}\text { No PAC } \\
(\mathrm{n}=11,640)\end{array}$ & $\begin{array}{c}\text { OR } \\
(95 \% \mathrm{CI})^{*}\end{array}$ & $P$ Value \\
\hline Mortality & $34.9 \%$ & $37.0 \%$ & $0.91(0.87-0.97)$ & .001 & $6.7 \%$ & $2.4 \%$ & $2.95(2.56-3.39)$ & $<.001$ \\
\hline MCS use & $39.0 \%$ & $25.8 \%$ & $1.84(1.73-1.94)$ & $<.001$ & $3.0 \%$ & $0.3 \%$ & $11.18(7.78-16.07)$ & $<.001$ \\
\hline AKI requiring hemodialysis & $9.8 \%$ & $6.1 \%$ & $1.66(1.51-1.84)$ & $<.001$ & $1.0 \%$ & $3.6 \%$ & $3.62(2.95-4.45)$ & $<.001$ \\
\hline Acute kidney injury & $56.4 \%$ & $48.6 \%$ & $1.37(1.30-1.44)$ & $<.001$ & $32.0 \%$ & $18.1 \%$ & $2.13(2.00-2.26)$ & $<.001$ \\
\hline Transfusion & $27.9 \%$ & $22.8 \%$ & $1.31(1.23-1.39)$ & $<.001$ & $13.3 \%$ & $5.6 \%$ & $2.57(2.34-2.83)$ & $<.001$ \\
\hline Intubation & $56.9 \%$ & $50.8 \%$ & $1.28(1.21-1.35)$ & $<.001$ & $10.0 \%$ & $2.1 \%$ & $5.14(4.47-5.91)$ & $<.001$ \\
\hline Vascular complications & $19.6 \%$ & $17.6 \%$ & $1.14(1.07-1.22)$ & $<.001$ & $5.9 \%$ & $1.6 \%$ & $3.84(3.26-4.52)$ & $<.001$ \\
\hline Acute respiratory failure & $45.9 \%$ & $43.4 \%$ & $1.11(1.05-1.17)$ & $<.001$ & $13.4 \%$ & $6.9 \%$ & $2.07(1.90-2.27)$ & $<.001$ \\
\hline Major Bleeding & $7.8 \%$ & $7.7 \%$ & $1.03(0.93-1.13)$ & .616 & $3.5 \%$ & $2.2 \%$ & $1.60(1.37-1.87)$ & $<.001$ \\
\hline LOS $\geq 5 \mathrm{~d}$ & $81.0 \%$ & $67.7 \%$ & $2.04(1.92-2.17)$ & $<.001$ & $75.6 \%$ & $43.0 \%$ & $4.10(3.88-4.34)$ & $<.001$ \\
\hline PCI & $19.0 \%$ & $22.4 \%$ & $0.81(0.76-0.87)$ & $<.001$ & $2.8 \%$ & $1.5 \%$ & $1.92(1.60-2.31)$ & $<.001$ \\
\hline Cardiac arrest & $15.1 \%$ & $18.9 \%$ & $0.76(0.71-0.81)$ & $<.001$ & $1.5 \%$ & $0.6 \%$ & $2.67(2.01-3.56)$ & $<.001$ \\
\hline Median LOS, d (IQR) & $12(6-20)$ & $8(3-15)$ & - & $<.001$ & $8(5-14)$ & $4(2-7)$ & - & $<.001$ \\
\hline Median hospital costs & $\$ 50,911$ & $\$ 31,734$ & - & $<.001$ & $\$ 20,168$ & $\$ 7,869$ & - & $<.001$ \\
\hline CABG & $15.0 \%$ & $13.2 \%$ & $1.16(1.08-1.25)$ & $<.001$ & $3.5 \%$ & $0.4 \%$ & $9.41(6.91-12.81)$ & $<.001$ \\
\hline LVAD placement & $4.5 \%$ & $1.4 \%$ & $3.27(2.73-3.91)$ & $<.001$ & $2.2 \%$ & $0.3 \%$ & $7.19(5.09-10.16)$ & $<.001$ \\
\hline Heart transplantation & $2.0 \%$ & $0.8 \%$ & $2.45(1.91-3.14)$ & $<.001$ & $2.1 \%$ & $0.3 \%$ & $8.54(5.80-12.56)$ & $<.001$ \\
\hline Nursing home discharge & $10.8 \%$ & $9.5 \%$ & $1.17(1.03-1.33)$ & .013 & $15.0 \%$ & $12.6 \%$ & $1.90(1.64-2.20)$ & $<.001$ \\
\hline
\end{tabular}

AKI, acute kidney injury; CABG, coronary artery bypass graft; IQR, interquartile range; MCS, mechanical circulatory support; PCI, percutaneous coronary intervention; LVAD, left ventricular assist device; LOS, length of stay. 
CI $1.22-1.31 ; P<.001$ ), AKI (OR 1.47, CI 1.42-1.53; $P<.001$ ), and need for dialysis (OR 1.52, CI 1.43-1.62; $P<.001)$ was also present for PAC use in patients with $\mathrm{CS}$, but it was much more modest.

In patients with $\mathrm{HF}$ without $\mathrm{CS}$, PAC was more frequently used in those with short-term MCS (OR 18.19, CI 16.92-19.56; $P<.001)$, durable left ventricular assist device (LVAD; OR 19.04, CI 17.20-21.08; $P<.001$ ), or heart transplantation (OR 13.24, CI 11.53-15.20; $P<.001)$. Similarly, PAC in CS was more frequently used in those with short-term MCS (OR 1.82, CI 1.75-1.89; $P<.001$ ) or undergoing durable LVAD (OR 3.42, CI $3.11-3.78 ; P<.001)$ or heart transplantation $(2.0 \%$ vs $0.5 \%$, OR 1.12 , CI 1.06-1.18; $P<.001)$. These factors likely contribute to the association of PAC with longer LOS for patients with HF (9 days [CI 5-16] vs 4 days [2-6] $[P<.001]$ compared with non-PAC HF) and CS (12 days $[6-20]$ vs $7[3-14][P<.001]$ compared with non-PAC CS). After using Poisson regression to compare LOS between cohorts, the stay remained longer in both cohorts with PAC $(P<.001)$. Similarly, hospital costs were higher in patients receiving PAC for both $\mathrm{HF}$ (\$23,266 vs $\$ 7,091$; $P<.001)$ and CS $(\$ 49,873$ vs $\$ 28,286 ; P<.001)$.

\section{Trends in PAC Use}

During the study period, there was an initial decline in the use of PAC in patients with HF from 8 per 1000 admissions in 2004 to 6 per 1000 admissions in 2007 (this finding has been reported elsewhere). ${ }^{6}$ Subsequently, there was a steady increase in PAC use to 12 per 1000 admissions in 2014 ( $P$ trend $<0.001$ ). However, in patients with CS, there has been a steep and steady decline in the use of PAC in patients with CS, from 123 per 1000 admissions in 2004 to 78 per 1000 admissions in 2014 ( $P$ trend $<0.001$; Fig. 1 ).

There has been an overall decrease in mortality in patients with diagnoses of both $\mathrm{HF}$ with and without CS throughout the study period. In 2004, mortality for patients with $\mathrm{HF}$ without $\mathrm{CS}$ was $16.8 \%$ in those receiving PAC and $3.9 \%$ in those without PAC. During the study years, the decline in mortality in HF patients without CS was more pronounced in those with PAC (57.7\% mortality reduction) than in those without PAC (25.6\% mortality reduction). For patients with $\mathrm{CS}$, mortality rates also declined during the study years $(P$ trend $<0.001$ for those with and without PAC); in 2004, the mortality rates for those with and without PAC were $49.4 \%$ and $47.9 \%$ respectively, and decreased similarly to $41.3 \%$ and $43.2 \%$ in 2007 . By the end of the study period, mortality for CS patients with PAC was $29.7 \%$ compared with $38.1 \%$ in those without it; this represents an absolute mortality reduction of $39.9 \%$ in those with PAC and $20.5 \%$ in those without it (Fig. 2).

\section{Discussion}

Previous studies evaluated the use of PAC in critically ill patients but not specifically in those with CS..$^{2,5,9}$ Our analysis demonstrates changing trends in use and outcomes with PAC for the diagnoses of $\mathrm{HF}$ with and without CS. CS remains the most common indication for PAC use, being used 10 times more frequently than in $\mathrm{HF}$ without $\mathrm{CS}$. However, its use in CS strikingly declined from 2004 to 2014, and this trend updates what was previously considered to be a modest rise. ${ }^{6}$ This practice change in patients with CS significantly differs from the patients with HF. As previously reported, ${ }^{3,7}$ there was an early decrease in the use of PAC in patients with HF from 1996 until 2007, when a subsequent rise in the use of PAC was seen; our study demonstrates that this rising trend has continued. More importantly, mortality in patients with CS and PAC has improved over time compared with those without PAC. This does not appear to be due solely to choosing healthier patients for PAC, because PAC use in HF without CS was associated with higher mortality $(9.9 \%$ vs $3.3 \% ; P<.001)$ although the excess mortality declined over time.

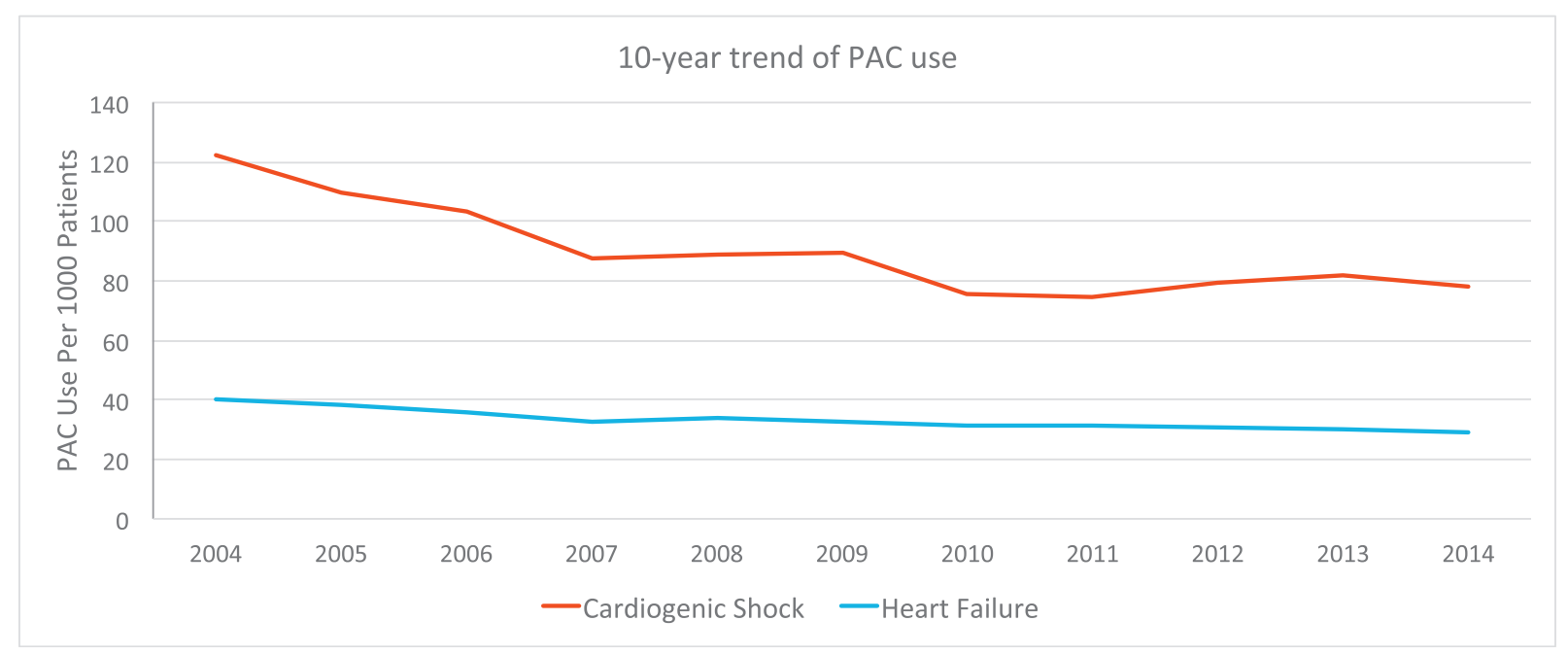

Fig. 1. Trends of pulmonary artery catheterization use in heart failure and cardiogenic shock. 


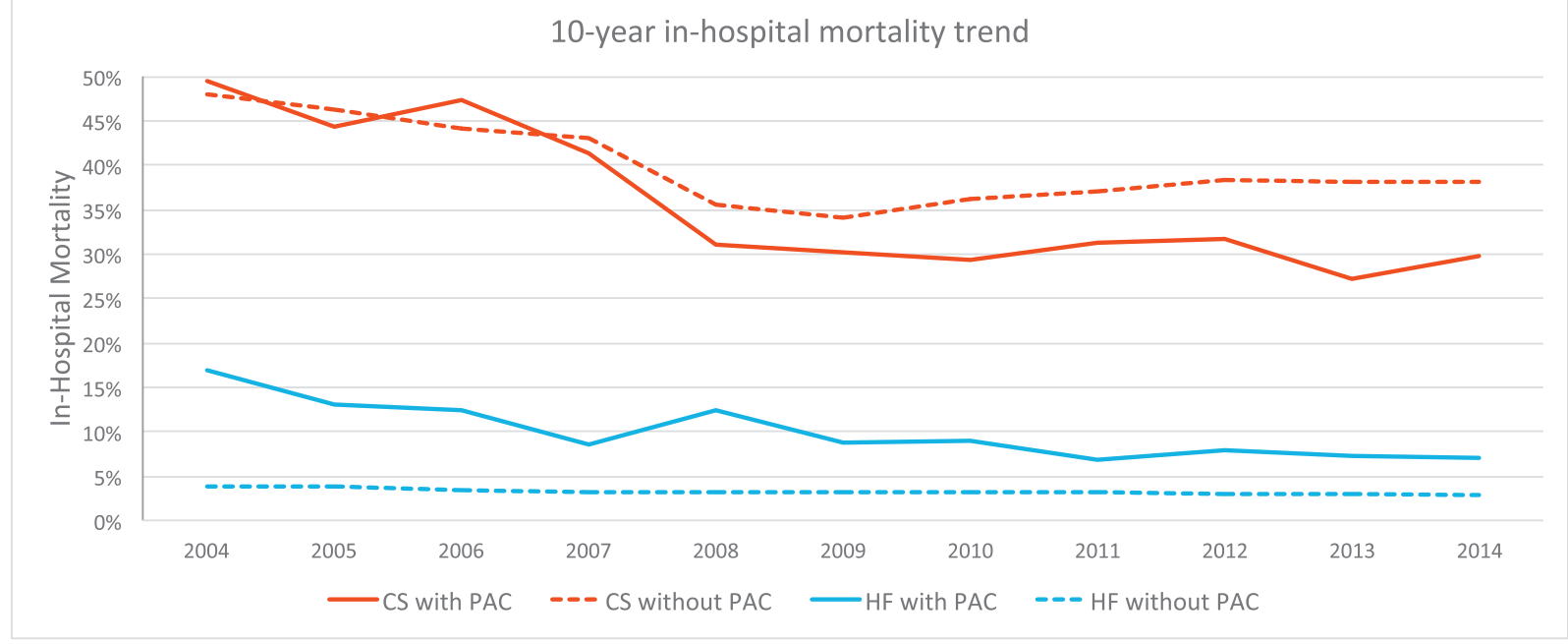

Fig. 2. Mortality in heart failure (HF) and cardiogenic shock (CS) with and without pulmonary artery catheterization (PAC) use.

During the past 3 decades, studies suggested that the PAC was not associated with benefits in a wide range of patients. ${ }^{1,5,9}$ The Study to Understand Prognoses and Preferences for Outcomes and Risks of Treatments (SUPPORT), ${ }^{9}$ showed that PAC was associated with increased mortality and resource utilization, leading to a recommendation for a moratorium on the use of PAC, although these outcomes were not seen in the subgroup of patients with HF. ${ }^{1}$ Analysis from the NIS during the time when those studies were carried out, from 1993 to 2004, reported a $65 \%$ decrease in PAC use in all medical admissions with a significant change in trend after early adoption of the SUPPORT results. ${ }^{3}$

In 2005, the ESCAPE investigators reported the first randomized controlled trial of PAC use in patients with severe symptomatic HF. ${ }^{2}$ This study recruited those sufficiently ill to make use of the PAC reasonable, but also sufficiently stable to minimize crossover. Patients with prior moderate doses of inotropic agents were excluded and the inotropic use for routine management of HF was discouraged. Patients with HF receiving a PAC could be enrolled in the ESCAPE registry if they failed to meet the eligibility criteria or the investigator perceived the PAC was necessary (62\% of the registry was perceived to be too sick for randomization). ${ }^{10}$ ESCAPE demonstrated that PAC did not affect the primary end point of days alive out of the hospital. In addition, registry patients had a higher mortality than those who underwent randomization..$^{10}$ ESCAPE was not designed to study CS patients in whom inotropic and vasopressor therapy are frequently used or for the assessment of potential MCS candidates, and in contrast to previous studies, it showed that PAC use appeared to be safe. Despite these neutral findings, there has been a readoption of PAC use in patients with HF, which has been attributed to the increased use of advanced HF therapies and increased prevalence of pulmonary hypertension and chronic kidney disease. ${ }^{6,7}$ Nonetheless, this readoption of the PAC has not occurred in patients with CS despite similar prevalence of these conditions.
Unlike previous NIS-based studies, we modified the methodology to not only include primary HF admissions, but also to gather those presenting with CS and no history of HF (45\% of patients in the CS group would otherwise have been excluded). Moreover, we did not exclude those with shortterm MCS, because we think that this intervention may be a reaction to the hemodynamic parameters obtained via PAC. Similarly to previous reports, patients with HF who received PAC were younger with fewer comorbidities such as ESRD, diabetes, and COPD, but they had higher rates of in-hospital death and cardiac arrest. Although baseline characteristics appeared to be more favorable, it is likely that the acuity of disease, not captured in depth in the NIS database, may account for their higher mortality. The increased use of PAC in patients in this cohort with complicating conditions such as respiratory failure, mechanical ventilation, and worsening renal function was also seen in the ESCAPE registry and aligns with current guideline recommendations for PAC. ${ }^{11}$ In other words, PAC may have identified HF patients who had a more critical condition with a higher mortality rate, albeit the excess mortality declined over time in our study. Using that premise, when analyzing those with CS, who also were younger and with fewer comorbidities, and assuming hospital practices were consistent during the study period, we found an intriguing paradox: patients with CS receiving PAC had a lower rate of in-hospital cardiac arrest and lower total mortality with a more significant decline over time. To be more precise, since 2007 the mortality trend for CS with and without PAC separated. This is the same time of the readoption of PAC in $\mathrm{HF}$ and the time when mortality started declining for those with HF and PAC. We think that this improvement in survival in those receiving PAC may be due in part to the advances of HF therapy and to the adoption of MCS. ${ }^{12}$ In 2005, the American College of Cardiology/American Heart Association guidelines ${ }^{13}$ recommended advanced treatment strategies such as mechanical circulatory support in those with stage D HF. In 2006, the Interagency Registry for Mechanically Assisted Circulatory Support 
(INTERMACS) ${ }^{14}$ started collecting data of patients receiving durable MCS, and within the first 18 months reported more than 400 patients supported with a durable device: $44.2 \%$ categorized as critical CS (profile I) and $35.2 \%$ categorized as declining despite inotrope escalation (profile II). By the end of our study period, 15,745 Food and Drug Administration-approved durable MCS devices were used, of which $52.5 \%$ were done in patients of INTERMACS profiles I and II; ${ }^{15}$ along with this, more than 4500 short-term devices had been used. ${ }^{12}$ In both HF and CS, PAC more frequently identified patients who underwent durable and shortterm devices. A recent study by Strom et $\mathrm{al}^{12}$ found an increased use of MCS for CS during the same period of time, yet they showed that mortality in CS has improved in both MCS and non-MCS groups. In addition, Nalluri et al, ${ }^{16}$ using the same database, found that in patients treated with the use of percutaneous MCS for post-myocardial infarction CS, the use of PAC was associated with improved outcomes. We hypothesize that better selection of patients for PAC and better use of the information to guide therapies is responsible for improved outcomes.

A recent scientific statement from the American Heart Association recommends the use of PAC in cases of diagnostic or CS management uncertainty or in patients who are unresponsive to initial therapy. ${ }^{17}$ Only one of the studies used in this consensus statement favors the use of PAC in patient with CS; the study by Rosello et $\mathrm{al}^{18}$ reported a single center analysis with 129 patients admitted with CS, and found that PAC was associated with lower short-term mortality (hazard ratio 0.55 , CI $0.35-0.86 ; P=.008$ ); our analysis extends this finding to a nationally representative cohort.

Finally, the mainstay intervention in CS should be that of early diagnosis and reverting the downward spiral of progressive myocardial dysfunction by selecting pharmacologic and mechanical support to maintain adequate tissue perfusion. ${ }^{17,19}$ To date there are no large-scale trials studying the benefit of PAC in CS. Even if one were designed, the perceived urgency of the need for information to guide decisions would undermine equipoise and enrollment for the sickest patients. In the absence of trials, the latest scientific statement recommends the use of PAC in this population, and the present analysis, the largest to date, supports this recommendation.

\section{Study Limitations}

The limitations of our study partly relate to the observational analysis of a large administrative database using ICD-9-CM codes, which may carry possible coding errors, changes in coding practices, underreporting of secondary or comorbid diagnoses or even overcoding (reducing the severity of CS diagnoses). The NIS lacks clinical details such as information about hemodynamic parameters, severity of HF (eg, ejection fraction, HF stage, New York Heart Association functional classification, or INTERMACS profiles), use of inotropic or vasoactive agents, and laboratory markers. Furthermore, we may have underestimated the true frequency of PAC use because we were unable to capture those performed but not coded at hospital discharge or those diluted when more than 15 procedures were coded (which, accounted for only $2.6 \%$ of our cohort). We also lacked long-term follow-up data, and we could determine in-hospital events only for a particular admission. Nonetheless, our study is a contemporary analysis of a large nationwide sample size with comprehensive analysis and highlights the impact of PAC in patients with CS.

\section{Conclusion}

This study demonstrates that although the use of PAC has decreased in patients with CS, its use is now associated with improved outcomes, which may reflect better selection of patients or better use of information to guide therapies. Our data provide reassurance that PAC use in this population is an appropriate strategy. In addition, there have been major advances in medical and device therapies for HF since the ESCAPE trial was released. These advances have come with readoption of the PAC and even though mortality for HF without CS remains higher in patients who have been selected for PAC, their mortality decreased over our the study period, reflecting concurrent progress in their selection and management.

\section{Disclosures}

J.L.: consultant: Novartis, Edwards, Abbott, Relypsa, Resmed, VWave; grants: National Institutes of Health, American Heart Association (AHA), Novartis, Astra Zeneca.

Remaining authors: None.

\section{Supplementary Data}

Supplementary data related to this article can be found at doi:10.1016/j.cardfail.2019.03.004.

\section{References}

1. Dalen JE, Bone RC. Is it time to pull the pulmonary artery catheter? JAMA 1996;276:916-8.

2. Binanay C, Califf RM, Hasselblad V, et al. Evaluation study of congestive heart failure and pulmonary artery catheterization effectiveness: the ESCAPE trial. JAMA 2005;294:1625-33.

3. Wiener RS, Welch HG. Trends in the use of the pulmonary artery catheter in the United States 1993-2004. Jama 2007; 298:423-9.

4. Shah MR, Hasselblad V, Stevenson LW, et al. Impact of the pulmonary artery catheter in critically ill patients: meta-analysis of randomized clinical trials. JAMA 2005;294:1664-70.

5. Harvey S, Harrison DA, Singer M, et al. Assessment of the clinical effectiveness of pulmonary artery catheters in management of patients in intensive care (PAC-Man): a randomised controlled trial. Lancet 2005;366(9484):472-7.

6. Pandey A, Khera R, Kumar N, Golwala H, Girotra S, Fonarow GC. Use of pulmonary artery catheterization in US patients with heart failure 2001-2012. JAMA Intern Med 2016; 176:129-32. 
7. Khera R, Pandey A, Kumar N, et al. Variation in hospital use and outcomes associated with pulmonary artery catheterization in heart failure in the United States. Circ Heart Fail 2016;9(11):e003226.

8. Ikuta K, Wang Y, Robinson A, Ahmad T, Krumholz HM, Desai NR. National trends in use and outcomes of pulmonary artery catheters among Medicare beneficiaries 1999-2013. JAMA Cardiol 2017;2:908-13.

9. Connors AF, Speroff T, Dawson N V. SUPPORT Investigators. The effectiveness of right heart catheterization in the initial care of critically ill patients. JAMA 1996;276:889-97.

10. Allen LA, Rogers JG, Warnica JW, et al. High mortality without ESCAPE: the registry of heart failure patients receiving pulmonary artery catheters without randomization. J Card Fail 2008; 14:661-9.

11. Yancy CW, Jessup M, Bozkurt B, et al. 2013 ACCF/AHA guideline for the management of heart failure: executive summary: a report of the American College of Cardiology Foundation/American Heart Association Task Force on Practice Guidelines. J Am Coll Cardiol 2013;62:1495-539.

12. Strom JB, Zhao Y, Shen C, et al. National trends, predictors of use, and in-hospital outcomes in mechanical circulatory support for cardiogenic shock. EuroIntervention 2018;13:e2152-9.

13. Hunt SA. American College of Cardiology. American Heart Association Task Force on Practice Guidelines (Writing Committee to Update the 2001 Guidelines for the Evaluation and
Management of Heart Failure). ACC/AHA 2005 guideline update for the diagnosis and management of chronic heart failure in the adult: a report of the American College of Cardiology/American Heart Association Task Force on Practice Guidelines (Writing Committee to Update the 2001 Guidelines for the Evaluation and Management of Heart Failure). J Am Coll Cardiol 2005;46:e1-82.

14. Kirklin JK, Naftel DC, Stevenson LW, et al. INTERMACS database for durable devices for circulatory support: first annual report. J Heart Lung Transplant 2008;27:1065-72.

15. Kirklin JK, Naftel DC, Pagani FD, et al. Seventh INTERMACS annual report: 15,000 patients and counting. J Heart Lung Transplant 2015;34:1495-504.

16. Nalluri N, Patel NJ, Atti V, Kumar V, Basir MB, O'Neill WW. Temporal trends in utilization of right-sided heart catheterization among percutaneous ventricular assist device recipients in acute myocardial infarction complicated by cardiogenic shock. Am J Cardiol 2018;122:2014-7.

17. van Diepen S, Katz JN, Albert NM, et al. Contemporary management of cardiogenic shock: a scientific statement from the American Heart Association. Circulation 2017;136:e232-68.

18. Rossello X., Vila M., Rivas-Lasarte M., et al. Impact of pulmonary artery catheter use on short- and long-term mortality in patients with cardiogenic shock. Cardiology 2017;136(1):61-69.

19. Hollenberg SM, Kavinsky CJ, Parrillo JE. Cardiogenic shock. Ann Intern Med 1999;131:47-59. 